BJMG $19(1), 2016 \cdot 5-12$

DOI: $10.1515 /$ bjmg-2016-0001

ORIGINAL ARTICLE

\title{
EMBRYO QUALITY PREDICTIVE MODELS BASED ON CUMULUS CELLS GENE EXPRESSION
}

\author{
Devjak R ${ }^{1}$, Burnik Papler T ${ }^{2}$, Verdenik I ${ }^{2}$, Fon Tacer $\mathrm{K}^{3}$, Vrtačnik Bokal E²
}

*Corresponding Author: Rok Devjak, M.D., Ph.D., Division of Medical Oncology, Institute of Oncology, Zaloška 2, 1000 Ljubljana, Slovenia. Tel: +386-1-5879-282; Fax: +386-1-5879-303. E-mail: rdevjak@onko-i.si

\begin{abstract}
Since the introduction of in vitro fertilization (IVF) in clinical practice of infertility treatment, the indicators for high quality embryos were investigated. Cumulus cells (CC) have a specific gene expression profile according to the developmental potential of the oocyte they are surrounding, and therefore, specific gene expression could be used as a biomarker. The aim of our study was to combine more than one biomarker to observe improvement in prediction value of embryo development. In this study, $58 \mathrm{CC}$ samples from 17 IVF patients were analyzed. This study was approved by the Republic of Slovenia National Medical Ethics Committee. Gene expression analysis [quantitative real time polymerase chain reaction (qPCR)] for five genes, analyzed according to embryo quality level, was performed. Two prediction models were tested for embryo quality prediction: a binary logistic and a decision tree model. As the main outcome, gene expression levels for five genes were taken and the area under the curve (AUC) for two prediction models were calculated. Among tested genes, AMHR 2 and LIF showed significant expression difference between high quality and low quality embryos. These two genes were used for the
\end{abstract}

\footnotetext{
$\overline{{ }^{1} \text { Division of Medical Oncology, Institute of Oncology, Ljubljana, }}$ Slovenia

${ }^{2}$ Reproductive Unit, Department of Obstetrics and Gynaecology, University Medical Centre Ljubljana, Ljubljana, Slovenia

${ }^{3}$ Faculty of Medicine, University of Ljubljana, Ljubljana, Slovenia
}

construction of two prediction models: the binary logistic model yielded an AUC of $0.72 \pm 0.08$ and the decision tree model yielded an AUC of $0.73 \pm$ 0.03 . Two different prediction models yielded similar predictive power to differentiate high and low quality embryos. In terms of eventual clinical decision making, the decision tree model resulted in easy-tointerpret rules that are highly applicable in clinical practice.

Keywords: $A M H R 2$ gene; Cumulus cells $(\mathrm{CC})$; Embryo prediction; LIF gene, In vitro fertilization (IVF)

\section{INTRODUCTION}

Since the introduction of in vitro fertilization (IVF) in the clinical practice of infertility treatment, indicators of implantation potential of embryos have been researched. Despite significant improvements in assisted reproductive technology (ART), the success of IVF remains low. Although most of the oocytes retrieved after ovarian stimulation with gonadotropins in combination with gonadotro-pin-releasing hormone $(\mathrm{GnRH})$ analogues are capable of fertilization, only half of them develop into embryos and even fewer implant [1]. Therefore, to increase pregnancy rate, more than one embryo is usually transferred, which can lead to multiple pregnancies and increased fetal and maternal morbidity and mortality [2]. Consequently, there is a need for identifying biomarkers that would serve as reliable indicators of high implantation potential of the embryos available for transfer [3]. 
The selection of embryos for transfer is currently based on the evaluation of subjective morphological criteria. These include evaluation of pronuclei in the zygote and early cleavage. On day 3 after oocyte retrieval, the embryo fragmentation, number and morphology of blastomeres, and the presence of multiple nuclei are evaluated; on day 5, blastocyst morphology is evaluated considering the Gardner and Schoolcraft system [4]. The presence of a mature (MII) and highquality oocyte plays an essential role in the development of a high-quality embryo [5]. This means that the selection of high-quality embryos begins at the time of oocyte selection. The oocyte selection for fertilization is currently also based on morphological evaluation of the polar body, meiotic spindle, zona pellucida and cytoplasm [6]. There is increasing evidence that morphological evaluation is not a reliable predictor of oocyte competence and embryo implantation potential [7]; that is why there is a need to discover new, noninvasive, objective and reliable indicators of oocyte and embryo quality. Having reliable biomarkers for oocyte and embryo selection could be of special importance in selective embryo transfer to avoid a twin pregnancy. Lately, the most intense research is being carried out on genome analysis of cumulus cells (CC) and granulosa cells (GC) in order to discover biomarkers that would be predictive of oocyte and embryo developmental potential [8-10].

It is well known that there is intense bidirectional communication between oocytes and their surrounding CC and GC through gap junctions and paracrine signaling during folliculogenesis [11]. This communication is crucial for the development of a mature, developmentally competent oocyte. Instead of being a passive recipient of nutrients and regulatory signals from its surrounding $\mathrm{CC}$ and $\mathrm{GC}$, the oocyte plays an active role in the secretion of paracrine factors that maintain an appropriate micro environment for the acquisition of its developmental competence [12]. This leads to functional changes in CC and GC which are crucial for the development of a quality oocyte [13]. In clinical practice, this means that these cells can serve as an indirect marker of oocyte quality. In IVF procedures, these cells are separated from oocytes and then discarded. They are easily accessible and plentiful, which makes them a perfect material for gene expression analysis in order to identify reliable and objective biomarkers of oocyte quality and embryo development potential [11].
Cumulus cells have been the subject of many studies in order to test whether oocyte quality is related to the expression of some of the growth differentiation factor 9 (GDF9)-dependent genes (HAS2, PTGS2 in PTX3) $[3,14,15]$. Furthermore, CC have been analyzed in terms of gene expression related to the quality of embryo development. van Montfoort et al. [9] proposed a set of the following genes: $C C N D 2$, CXCR4, GPX3, CTNND1, DHCR7, DVL3, HSPB1 and TRIM28 that have proven to be most variably expressed among the $\mathrm{CC}$ of the follicles with zygotes that underwent a rapid division, and the $\mathrm{CC}$ of those follicles the zygotes of which underwent a slow division [9]. Hamel et al. [16] proposed the following set of genes: FDX1, CYP19A1, CDC42, SERPINE2 and $3 \beta H S D 1$ as those having the most variable expression among the GC from the follicles that resulted in pregnancy and those that did not.

In our previous study [10], we identified CC expression of AMHR2, LIF, SERPINE2, VEGFC and $F S H R$ to be associated with blastocyst formation. In that study, LIF did not pass correction for multiple hypothesis testing, but due to its previous implication for oocyte maturation [17], we included it in our further analyses of CC expression. In this study, we used these genes to construct an embryo quality outcome model according to $\mathrm{CC}$ gene expression from oocytes that resulted in either high or low quality embryos.

\section{MATERIALS AND METHODS}

Patients and In Vitro Fertilization Treatment. In this study, 17 patients undergoing the classical IVF cycle at the Department of Obstetrics and Gynecology, University Medical Centre, Ljubljana, Slovenia, were included. The study was approved by the Republic of Slovenia National Medical Ethics Committee (http://www.kme-nmec. si/) and patients signed a written consent form prior to study inclusion. It included patients who were less than 35 years old and with body mass index (BMI) between 17 and $26 \mathrm{~kg} / \mathrm{m}^{2}$. They attended the IVF program because of tubal factor infertility. The spermiograms of their partners were normal, according to the World Health Organization (WHO) criteria.

As our previous study did not expose any differences in $\mathrm{CC}$ gene expression between patients who were treated with either GnRH agonists or antagonists in combination with recombinant follicle-stimulating 
hormone (rFSH) [10], we used both GnRH analogs in the present study. Ten patients were administered GnRH agonist buserelin acetate (Suprefact; Hoechst AG, Frankfurt/Main, Germany) starting from day 22 with a daily dose of $0.6 \mathrm{ml}(600 \mathrm{pg})$ subcutaneously. When criteria for ovarian desensitization were fulfilled (eastradiol $<0.05 \mathrm{nmol} / \mathrm{L}$, follicles $<5 \mathrm{~mm}$ in diameter), patients were subcutaneously administered 225 IU of gonadotropin folitropin $\alpha$ (Gonal F; Industria Farmaceutica Serono S.p.A, Bari, Italy). The other seven patients received 225 IU of gonadotropin folitropin $\alpha$, subcutaneously administered on day 2 . When the dominant follicle measured $\geq 14 \mathrm{~mm}$ in diameter, the $\mathrm{GnRH}$ antagonist cetrorelix acetate (Cetrotide; Asta Medica AG, Frankfurt, Germany) in a dose of $0.25 \mathrm{mg}$, was administered subcutaneously.

Afterwards, all patients received 10,000 IU of the human chorionic gonadotropin (hCG) (Pregnyl; N.V. Organon, Oss, the Netherlands) when at least three follicles were $\geq 17 \mathrm{~mm}$ and serum oestradiol was $\geq 0.40 \mathrm{nmol} / \mathrm{L}$ per follicle; $34-36$ hours later, ultrasound-guided transvaginal oocyte retrieval was performed.

Cumulus Cells Collection and Oocyte FollowUp. Oocytes were removed from the follicular fluid. Immediately after oocyte retrieval, a small sample of $\mathrm{CC}$ of each oocyte was removed using a needle and a glass denudation pipette (Swemed, Göteborg, Sweden). Oocytes were not denuded by this technique. Obtained CC samples were washed in phosphatebuffered saline (PBS), snap frozen in liquid nitrogen and stored at $-80^{\circ} \mathrm{C}$ in vials until RNA isolation.

The oocytes were further inseminated (classical IVF) and cultivated individually. After 24 hours, oocyte fertilization status was assessed. Fertilized oocytes were further cultured to the blastocyst stage in the Universal IVF Medium followed by the BlastAssist System (M1 and M2; Origio, Målov, Denmark) for 5 days. On day 5, at most two embryos at the blastocyst or morula stage were transferred into the uterus. Supernumerary blastocysts were cryopreserved.

Experimental Design. The models were built on the CC expression level values of five genes (AMHR2, LIF, SERPINE2, VEGFC and FSHR) of two kinds of embryos: high quality embryos $(n=26)$, represented by morula and blastocyst stage embryos on day 5 , and low quality embryos $(n=36)$, represented by embryos which arrested in development any time within 5 days of cultivation after fertiliza- tion. The decision trees probabilistic model was used to estimate sensitivity, specificity and area under the curve (AUC) of a proposed model.

Quantitative Real Time Polymerase Chain Reaction Analysis. Quantitative real time PCR (qPCR) was used for $\mathrm{CC}$ gene expression using TaqMan Gene Expression pre designed assays (Applied Biosystems, Foster City, CA, USA). Peptidylprolyl isomerase B $(P P I B)$ and $18 \mathrm{~s}$ rRNA were added for normalization. Genomic DNA contamination was eliminated by DNAse treatment using DNAse I (F. Hoffmann-La Roche Ltd., Basel, Switzerland). cDNA for qPCR assays was prepared from $200 \mathrm{ng}$ DNAsed RNA using SuperScript RT III (Invitrogen, Carlsbad, CA, USA) in a final volume of $20 \mu \mathrm{L}$. Following cDNA synthesis, RNAse-free water was added to increase the sample volume to $30 \mu \mathrm{L}$. Measurements were performed using a LightCycler 480 System (Roche Applied Science, Penzberg, Germany). Normalized mRNA levels were obtained by dividing the averaged, efficiency corrected values for mRNA expression by a normalization factor calculated from peptidylprolyl isomerase B (PPIB) and 18 s rRNA values and are expressed in arbitrary units. The resulting values were $\log 2$ transformed (log2-fold change) for comparison with microarray data.

\section{Statistical Analysis and Decision Tree Model}

Construction. A Mann-Whitney U test and logistic regression model were performed with the Statistical Package for the Social Sciences (SPSS) version 17.0 (SPSS Inc., Chicago, IL, USA). For the decision tree model construction Orange $\AA$ (www.orange.biolab.si) was used. Orange ${ }^{\circledR}$ is a data mining tool that works through preprogrammed widgets. Designing and testing decision trees is one of its functions.

\section{RESULTS}

The baseline characteristics between the two embryo groups (high quality vs. low quality) were not altered significantly by age, BMI and patients' distribution tests in a binary logistic regression model (Table 1). The pregnancy rate for the observed group of patients was 0.53 and delivery rate was 0.47 . Because RNA level from four CC samples of low quality embryos was to low, only $32 \mathrm{CC}$ samples were analyzed, therefore, $58 \mathrm{CC}$ samples were included in the predictive model construction. The Mann-Whitney U test showed significant difference between $\mathrm{CC}$ gene expression of oocytes resulting in high quality and 
Table 1. Binary logistic model for embryo outcome where age, body mass index and patient distribution were tested between high quality and low quality embryo groups. None of the tested variables were shown to be significant in our data.

\begin{tabular}{|l|c|c|c|c|}
\hline Parameters & Significance $^{\mathrm{a}}$ & $\mathbf{E x p}(\mathbf{B})$ & \multicolumn{2}{|c|}{ 95\% CI for $\operatorname{Exp}(\mathbf{B})$} \\
\hline & & & Lower & Upper \\
\hline Age & 0.26 & 1.10 & 0.93 & 1.28 \\
\hline Body mass index & 0.82 & 0.98 & 0.79 & 1.21 \\
\hline Patient & 0.25 & 0.93 & 0.82 & 1.05 \\
\hline Constant & 0.67 & & & \\
\hline
\end{tabular}

$\operatorname{Exp}(\mathrm{B})$ : expectancy for $\beta ; 95 \% \mathrm{CI}: 95 \%$ confidence interval.

a Significance in the model expressed as a $p$ value.

Table 2. The Mann-Whitney U test results for all analyzed genes between high quality and low quality embryos (Mann-Whitney $\mathrm{U}$ test column); furthermore, data were split into AMHR2 high CC expression and low CC expression and analyzed using the Mann-Whitney $\mathrm{U}$ test (AMHR2 high group and AMHR2 low group columns).

\begin{tabular}{|c|c|c|c|}
\hline Gene & $\begin{array}{l}\text { Mann-Whitney } \mathbf{U} \\
\text { (significance) }^{\mathrm{a}}\end{array}$ & $\begin{array}{c}\text { AMHR2 High Group } \\
\text { (significance) }^{\mathrm{a}}\end{array}$ & $\begin{array}{c}A M H R 2 \text { Low Group } \\
\text { (significance) }^{\mathrm{a}}\end{array}$ \\
\hline AMHR2 & 0.03 & - & - \\
\hline FSHR & 0.44 & 0.52 & 0.09 \\
\hline$L I F$ & 0.11 & 0.03 & 0.59 \\
\hline SERPINE2 & 0.74 & 0.72 & 0.37 \\
\hline 0.72 VEGFC & 0.72 & 0.34 & 0.75 \\
\hline
\end{tabular}

a Significance expressed as a $p$ value.

low quality embryos only in $A M H R 2(p=0.030)$. The data were then split according to $A M H R 2$ expression (high or low, where the median was the cut-off value) and genes were further tested in each group (Table 2). In the $A M H R 2$ gene, high expression group $L I F$ was shown to differ significantly between high quality and low quality embryos $(p=0.033)$. Therefore, $A M H R 2$ and $L I F$ were taken for the construction of the embryo quality outcome prediction model.

Binary Logistic Regression Model. The AMHR2 and LIF CC expression values were used to construct three different binary logistic regression models for predicting a high quality embryo outcome. First, $A M H R 2$ and LIF CC expression values were used in separate models, and their prediction values yielded an AUC of $0.69 \pm 0.08$ and $0.63 \pm 0.08$, respectively. Then, both genes CC expression values were combined into one model in which the prediction value yielded an AUC of $0.72 \pm 0.08$.

Decision Tree Model. The same procedure was used in a data mining protocol for constructing three decision trees with $A M H R 2$ and $L I F$ CC expression values. A simple data discretization was used for node splitting in the decision tree where expression values were stratified into two equal frequency intervals (high and low CC expression values). The decision tree model was tested using $50.0 \%$ of the data for learning and $50.0 \%$ data for testing. Testing was then repeated 100 times; median AUC and standard deviation were calculated. First, $A M H R 2$ and $L I F \mathrm{CC}$ expression values were used to construct separate decision tree models, and their prediction values yielded an AUC of $0.67 \pm 0.01$ and $0.57 \pm 0.02$, respectively. Combining both genes resulted in a decision tree (Figure 1) with an AUC of $0.73 \pm 0.03$.

\section{DISCUSSION}

In the present study, we analyzed CC expression of AMHR2, LIF, SERPINE2, VEGFC and FSHR from oocytes that developed into either high quality or low quality embryos. In our previous study [10], selected genes were shown to be well differentiated between immature MI and mature MII oocytes, according to $\mathrm{CC}$ expression. In this study, we use them as potential biomarkers of embryo quality. Only AMHR 2 and $L I F$ were shown to be significant and were used in our prediction models. In either the binary logistic model or decision tree model, the predictive power was the best when both genes were used simultaneously. 


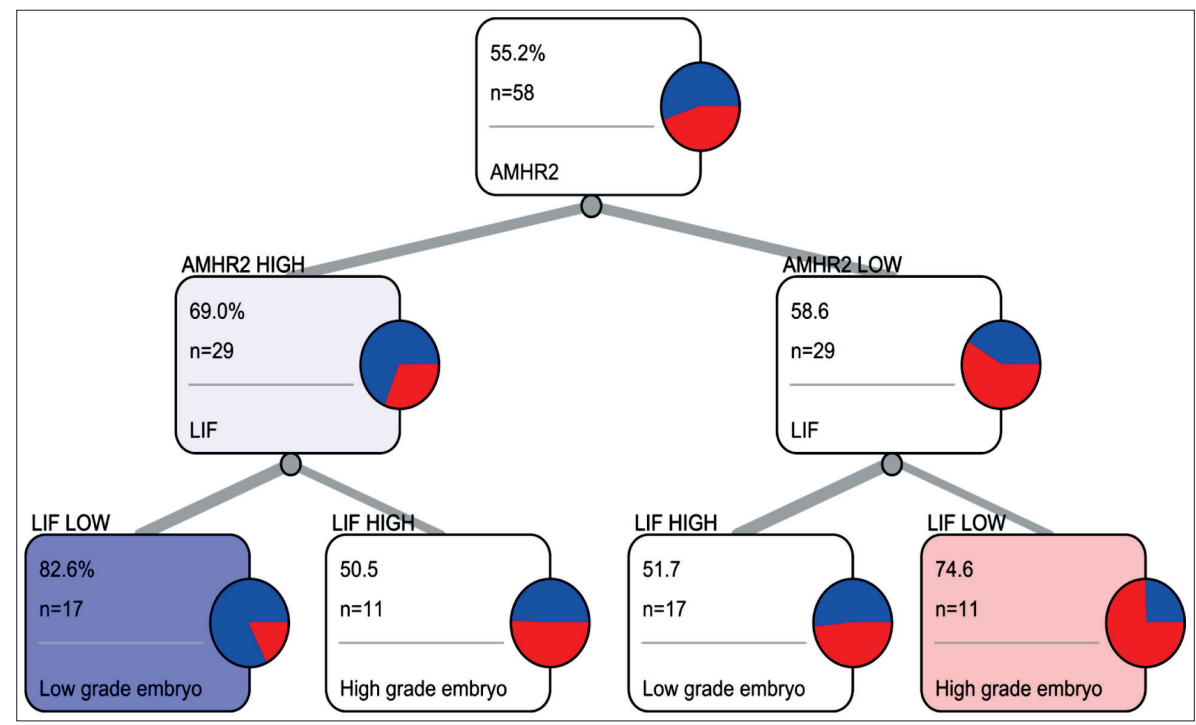

Figure 1. A decision tree model diagram with $A M H R 2$ and LIF. The model first separates $\mathrm{CC}$ samples upon $A M H R 2$ expression (high or low) and then upon $L I F$ expression (high or low). The blue color represents leaves with predominantly CC of low quality embryos and red color represents leaves with predominantly $\mathrm{CC}$ of high quality embryos. A combination of high AMHR2 and low LIF CC expression leads to an $82.6 \%$ possibility of developing a low quality embryo, and combination of low $A M H R 2$ and low LIF CC expression leads to $74.6 \%$ possibility of developing high quality embryos.

In recent years, many studies have been performed to analyze $\mathrm{CC}$ gene expression in association with various endpoints: oocyte maturity, embryo development and pregnancy $[8,9,16,18]$. Since $C C$ is an easily accessible material that is normally discarded during the IVF cycle, it represents a good biological material for research and hopefully, someday, also for diagnostic purposes. In previous studies on $\mathrm{CC}$ gene expression, many genes were shown to be differentially expressed between observed groups of oocytes, but not many were tested for predictive power [19]. As a main characteristic of a good diagnostic test is high predictive power, the AUC value (defined by high sensitivity and specificity), only genes which would yield a good predictive power, whether alone or in combination with other genes in repeated trials, would be suitable for potential CC gene expression diagnostic testing.

The AMH works through its receptor AMHR2, being the highest in the preantral follicle for their recruitment, and during follicle maturation it gradually diminishes [20-22]. It has also been proved that AMHR2 CC level decreases with the level of oocyte maturity and in the same manner is expressed by $A M H$ in CC $[10,23]$. In this study, the CC expression of $A M H R 2$ was significantly negatively correlated with embryo quality. This again indicates that oocyte maturity is a prerequisite for high quality embryo development. In this study two models were constructed with $A M H R 2$ for high quality embryo prediction, and both showed similar predictive power. In comparison to models based on LIF CC expression, AMHR2based models show better predictive power, which can be well-explained by higher $\mathrm{CC}$ expression differences between high and low quality embryos in $A M H R 2$ compared to $L I F$.

The connection of LIF with reproduction was shown in the study where LIF was abundantly expressed in the uterine endometrial glands on day 4 of pregnancy [24]. Namely, the $\mathrm{p} 53$ protein regulates the $L I F$ expression and sufficient LIF levels are crucial for embryo implantation $[25,26]$. In addition, the role of LIF in reproduction is not only in implantation but also in CC expansion. In the study of LIF function in in vitro maturated human and mice cumulus-oophorus complex, it was proven that LIF supple-mentation induced cumulus expansion in both settings [17]. LIF also plays a role in blastocyst formation, where the group of bovine cumulus-oocyte complexes that were incubated with LIF yielded higher blastocyst development compared to the control group without LIF [27]. In our study LIF CC expression showed sig- 
nificance between $\mathrm{CC}$ of high quality and low quality embryos only when a subgroup with high $A M H R 2$ $C C$ expression was observed. Therefore, using $L I F$ alone in the model results in lower predictive power than $A M H R 2$ but when used together, LIF improves the prediction value of $A M H R 2$. Logistic regression represents the gold standard for constructing prediction models in biomedical studies. As its statistic is based on logistic regression, for each attribute the model computes a coefficient and combines them into one prediction variable. These are used for the receiver-operator curve (ROC) and computing AUC values, but as such, a prediction variable has no informational value for to user (i.e. clinician). Predictive data mining has become one of the essential tools for the researcher in medicine [28]. One of these techniques is also the decision tree, where a decision is made in each node, according to the value and predictive power of the variable. At the end (leaves) the probability of an event is given. Decision trees are usually represented with diagrams but can also be with "if sentences." Diagrams make the decision tree model easy to interpret, therefore, a model with more informational value for the user.

In our study, both types of models, binary logistic and decision trees, resulted in similar AUC values, indicating that the type of the models used does not improve the predictive power. Analyzing the decision tree diagram of the model with $A M H R 2$ and $L I F$ leads to the conclusions that first, when both $A M H R 2$ and $L I F$ are low, there is a high possibility of the development of high quality embryos; second, when AMHR2 is high and $L I F$ is low, there is a high possibility of developing low quality embryos, and third, all other combinations of $A M H R 2$ and $L I F$ expression result in the equal possibility of developing of high quality or low quality embryos. An equal chance of developing a high quality or low quality embryo upon $A M H R 2$ and LIF expression actually means that the model is unable to predict the outcome, and this group contained exactly half of all observed embryos. Additional biomarkers would probably improve prediction for this group of embryos, or some other factors exist which we currently did not take into consideration, e.g. the quality of spermatozoa.

A logistic regression of CC expression was also used for constructing predictive models in the study by McKenzie et al. [29]. In their study, the expression values of hyaluronic acid synthase 2 (HAS2), cyclooxygenase 2 (PTGS2) and gremlin (GREM1) were used for constructing regression models for oocyte maturity, oocyte fertilization and embryo quality. Regression models for embryo quality yielded an AUC of 0.76, 0.76 and 0.81 for HAS2, PTGS2 and GREM1, respectively. Combining PTGS2 and GREM1 only slightly improved the predictive power (AUC 0.82 vs. 0.81). Besides PTGS2, the study by Wathlet et al. [30] also used six other genes and tested them for predictive power of cleavage stage embryo prediction and pregnancy prediction. Among the tested genes, the best cleavage stage embryo prediction relied on TRPM7 and ITPKA, but the AUC value was not calculated. Another prognostic model for pregnancy was published by lager et al. [31], where 12 genes previously recognized by microarray, were tested by qPCR for their predictive power. They used a "signal to noise" ratio to assess the predictive value of a gene using weighted voting. The AUC value for pregnancy prediction was $0.76 \pm 0.08$.

\section{CONCLUSIONS}

In this study, $A M H R 2$ correlated with high quality embryos, and its predictive power was higher when combined, with $L I F$. A set of highly predictive genes would probably result in a good prediction model where the decision tree model seems to have high clinical applicability.

Declaration of Interest. This study was supported by the Slovenian Research Agency (www. arrs.gov.si) grants L3-4162. The authors report no conflicts of interest. The authors alone are responsible for the content and writing of this article.

\section{REFERENCES}

1. Ferraretti AP, Goossens V, de Mouzon J, Bhattacharya S, Castilla JA, Korsak V, et al. Assisted reproductive technology in Europe, 2008: Results generated from European registers by ESHRE. Hum Reprod. 2013; 27(9): 2571-2584.

2. Keith L, Breborowicz G. Triplet pregnancies and their aftermaths. Part I: Basic considerations. Int J Fertil Womens Med. 2002; 47(6): 254-264.

3. Feuerstein P, Cadoret V, Dalbies-Tran R, Guerif F, Bidault R, Royere D. Gene expression in human cumulus cells: One approach to oocyte competence. Hum Reprod. 2007; 22(12): 3069-3077. 
4. Scott L. Pronuclear scoring as a predictor of embryo development. Reprod Biomed Online. 2003; 6(2): 201-214.

5. Sirard MA, Richard F, Blondin P, Robert C. Contribution of the oocyte to embryo quality. Theriogenology. 2006; 65(1): 126-136.

6. Scott $\mathrm{L}$. The biological basis of non-invasive strategies for selection of human oocytes and embryos. Hum Reprod Update. 2003; 9(3): 237-249.

7. Balaban B, Urman B. Effect of oocyte morphology on embryo development and implantation. Reprod Biomed Online. 2006; 12(5): 608-615.

8. Assou S, Anahory T, Pantesco V, Le Carrour T, Pellestor F, Klein B, et al. The human cumulus - Oocyte complex gene-expression profile. Hum Reprod. 2006; 21(7): 1705-1719.

9. van Montfoort AP, Geraedts JP, Dumoulin JC, Stassen AP, Evers JL, Ayoubi TA. Differential gene expression in cumulus cells as a prognostic indicator of embryo viability: a microarray analysis. Mol Hum Reprod. 2008; 14(3): 157-168.

10. Devjak R, Fon Tacer K, Juvan P, Virant Klun I, Rozman D, Vrtacnik Bokal E. Cumulus cells gene expression profiling in terms of oocyte maturity in controlled ovarian hyperstimulation using GnRH agonist or GnRH antagonist. PLoS One. 2012; 7(10): e47106. doi: 10.1371/ journal. pone. 0047106 .

11. Li R, Norman RJ, Armstrong DT, Gilchrist RB. Oocyte-secreted factor(s) determine functional differences between bovine mural granulosa cells and cumulus cells. Biol Reprod. 2000; 63(3): 839-845.

12. Matzuk MM, Burns KH, Viveiros MM, Eppig JJ. Intercellular communication in the mammalian ovary: Oocytes carry the conversation. Science. 2002; 296(5576): 2178-2180.

13. Assou S, Boumela I, Haouzi D, Anahory T, Dechaud H, De Vos J, et al. Dynamic changes in gene expression during human early embryo development: From fundamental aspects to clinical applications. Hum Reprod Update. 2010; 17(2): 272-290.

14. Cillo F, Brevini TA, Antonini S, Paffoni A, Ragni G, Gandolfi F. Association between human oocyte developmental competence and expression levels of some cumulus genes. Reproduction. 2007; 134(5): 645-650.
15. Zhang X, Jafari N, Barnes RB, Confino E, Milad M, Kazer RR. Studies of gene expression in human cumulus cells indicate pentraxin 3 as a possible marker for oocyte quality. Fertil Steril. 2005; 83(Suppl 1): 1169-1179.

16. Hamel M, Dufort I, Robert C, Gravel C, Leveille $\mathrm{MC}$, Leader A, et al. Identification of differentially expressed markers in human follicular cells associated with competent oocytes. Hum Reprod. 2008; 23(5): 1118-1127.

17. De Matos DG, Miller K, Scott R, Tran CA, Kagan D, Nataraja SG, et al. Leukemia inhibitory factor induces cumulus expansion in immature human and mouse oocytes and improves mouse two-cell rate and delivery rates when it is present during mouse in vitro oocyte maturation. Fertil Steril. 2008; 90(6): 2367-2375.

18. Assou S, Haouzi D, Mahmoud K, Aouacheria A, Guillemin Y, Pantesco V, et al. A non-invasive test for assessing embryo potential by gene expression profiles of human cumulus cells: A proof of concept study. Mol Hum Reprod. 2008; 14(2): 711-719.

19. Assou S, Haouzi D, De Vos J, Hamamah S. Human cumulus cells as biomarkers for embryo and pregnancy outcomes. Mol Hum Reprod. 2010; 16(8): 531-538.

20. Visser J. Role of anti-Müllerian hormone in follicle recruitment and maturation. J Gynecol Obstet Biol Reprod (Paris). 2006; 35(5 Pt 2): 2S30-2S4.

21. Weenen C, Laven JS, Von Bergh AR, Cranfield M, Groome NP, Visser JA, et al. Anti-Mullerian hormone expression pattern in the human ovary: Potential implications for initial and cyclic follicle recruitment. Mol Hum Reprod. 2004; 10(2): 77-83.

22. Andersen CY, Schmidt KT, Kristensen SG, Rosendahl M, Byskov AG, Ernst E. Concentrations of AMH and inhibin-B in relation to follicular diameter in normal human small antral follicles. Hum Reprod. 2010; 25(5): 1282-1287.

23. Grondahl ML, Nielsen ME, Dal Canto MB, Fadini R, Rasmussen IA, Westergaard LG, et al. AntiMullerian hormone remains highly expressed in human cumulus cells during the final stages of folliculogenesis. Reprod Biomed Online. 2011; 22(5 Pt 2): 389-398. 
24. Bhatt H, Brunet LJ, Stewart CL. Uterine expression of leukemia inhibitory factor coincides with the onset of blastocyst implantation. Proc Natl Acad Sci USA. 1991; 88(24): 11408-11412.

25. Lass A, Weiser W, Munafo A, Loumaye E. Leukemia inhibitory factor in human reproduction. Fertil Steril. 2001; 76(6):1091-1096.

26. Hu W, Feng Z, Teresky AK, Levine AJ. p53 Regulates maternal reproduction through LIF. Nature. 2007; 450(7170): 721-724.

27. Eswari S, Sai Kumar G, Sharma GT. Expression of mRNA encoding leukaemia inhibitory factor (LIF) and its receptor (LIFR $\beta$ ) in buffalo preimplantation embryos produced in vitro: Markers of successful embryo implantation. Zygote. 2013; 21(2): 203-213.

28. Bellazzi R, Zupan B. Predictive data mining in clinical medicine: Current issues and guidelines. Int J Med Inform. 2008; 77(2): 81-97.
29. McKenzie LJ, Pangas SA, Carson SA, Kovanci E, Cisneros P, Buster JE, et al. Human cumulus granulosa cell gene expression: A predictor of fertilization and embryo selection in women undergoing IVF. Hum Reprod. 2004; 19(12): 2869-2874.

30. Wathlet S, Adriaenssens T, Segers I, Verheyen G, Van de Velde H, Coucke W, et al. Cumulus cell gene expression predicts better cleavage-stage embryo or blastocyst development and pregnancy for ICSI patients. Hum Reprod. 2011; 26(5): 1035-1051.

31. Iager AE, Kocabas AM, Otu HH, Ruppel P, Langerveld A, Schnarr P, et al. Identification of a novel gene set in human cumulus cells predictive of an oocyte's pregnancy potential. Fertil Steril. 2013; 99(3): 745-752. 